\title{
Rough Mirror as a Quantum State Selector: Analysis and Design
}

\author{
M. Escobar, ${ }^{1}$ F. Lamy, ${ }^{2,3}$ A. E. Meyerovich, ${ }^{1}$ and V. V. Nesvizhevsky ${ }^{3}$ \\ ${ }^{1}$ Department of Physics, University of Rhode Island, Kingston, RI 02881-0817, USA \\ ${ }^{2}$ ICFP, Physics Department, École Normale Supérieure, 24 rue Lhomond, 75005 Paris, France \\ ${ }^{3}$ Institute Max von Laue-Paul Langevin, 38042 Grenoble, France \\ Correspondence should be addressed to A. E. Meyerovich; alexander_meyerovich@uri.edu
}

Received 14 May 2014; Accepted 23 June 2014; Published 4 August 2014

Academic Editor: Stefan Baessler

Copyright (C) 2014 M. Escobar et al. This is an open access article distributed under the Creative Commons Attribution License, which permits unrestricted use, distribution, and reproduction in any medium, provided the original work is properly cited. The publication of this article was funded by SCOAP S $^{3}$

\begin{abstract}
We report analysis of rough mirrors used as the gravitational state selectors in neutron beam and similar experiments. The key to mirror properties is its roughness correlation function (CF) which is extracted from the precision optical scanning measurements of the surface profile. To identify CF in the presence of fluctuation-driven fat tails, we perform numerical experiments with computergenerated random surfaces with the known CF. These numerical experiments provide a reliable identification procedure which we apply to the actual rough mirror. The extracted CF allows us to make predictions for ongoing GRANIT experiments. We also propose a radically new design for rough mirrors based on Monte Carlo simulations for the 1D Ising model. The implementation of this design provides a controlled environment with predictable scattering properties.
\end{abstract}

\section{Introduction}

One of the intriguing options for designing quantum state selectors which separate particles in desirable quantum states from all other particles is to use rough mirrors [1]. The idea is quite simple: the particles that reach the rough mirror are scattered away, while the particles locked in quantum states without an access to the rough mirror remain in the system.

Recent first experimental observation of gravitationally quantized ultracold neutrons (UCNs) [2-6] stimulated use of mirrors with random rough surfaces as state selectors instead of simple absorbers in beam and similar experiments with UCNs [7-9], as well as for shaping UCN spectra [1012] or exploring the whispering gallery quantum states of cold neutrons [13]. In these experiments a beam of UCNs with large horizontal and small vertical velocities propagates between two horizontal mirrors. The upper mirror is rough and serves as a state selector by scattering away the neutrons in the highest gravitational states which can reach it. The lower mirror is an ideal specular reflector for the neutrons. In the end, only the neutrons in the lowest gravitational states (neutrons with the lowest vertical velocities) remain in the system and continue bouncing along the lower mirror without scattering. Thus the absence of roughness is crucial for flat bottom mirrors $[14,15]$.

These types of selectors may have a wide range of applications and can be built not only for neutron beams, but also for other experiments with UCNs, ultracold atoms, and antiatoms [16-19], in particular within the GBAR project with ultracold antihydrogen at CERN [20], as well as, probably, with positronium [21] and so forth.

The ultimate resolution and precision for experiments of this kind with gravitational and whispering gallery quantum states of UCNs in the framework of GRANIT project [2224] benefits from the method of long storage of UCNs in closed quantum traps as well as from high UCN space-phase density in a dedicated UCN source [25]. More examples and details could be found in proceedings of dedicated GRANIT workshops [26, 27].

The ability of rough mirrors to serve as state selectors hinges on producing a desired pattern in scattering of particles by surface inhomogeneities. This scattering is determined by the correlation function of surface roughness (see, e.g., [28] and references therein). In beam experiments, scattering by the rough upper mirror turns the velocity vector thus increasing its vertical component. When the component of 
velocity normal to the mirrors exceeds a certain threshold velocity $v_{c}$, the neutrons can penetrate the mirror material and get absorbed; this threshold velocity can be translated into the absorption potential $U_{c}=m v_{c}^{2} / 2 \sim 10^{-26} \mathrm{~J}$. The roughness-driven transition probabilities between the quantum states $(j, \mathbf{q})$ and $\left(j^{\prime}, \mathbf{q}^{\prime}\right)$ for a mirror with slight roughness and the absorption threshold $U_{c}$ are [28]

$$
W_{j j^{\prime}}\left(\mathbf{q}, \mathbf{q}^{\prime}\right)=\zeta\left(\mathbf{q}-\mathbf{q}^{\prime}\right) U_{c}^{2}\left|\Psi_{j}(H)\right|^{2}\left|\Psi_{j^{\prime}}(H)\right|^{2}
$$

where $\mathbf{q}$ is the particle momentum along the wall, $\Psi_{j}(H)$ is the value of the wave function in quantum state $j$ on the mirror in the absence of roughness, and $\zeta(\mathbf{q})$ is the Fourier image of the correlation function of surface roughness (the so-called power spectrum). If the threshold is very high or the particle cannot penetrate the mirror material at all, $U_{c} \rightarrow \infty$, (1) becomes [28]

$$
W_{j j^{\prime}}=\frac{1}{4 m^{2}} \zeta\left(\mathbf{q}-\mathbf{q}^{\prime}\right)\left|\Psi_{j}^{\prime}(H)\right|^{2}\left|\Psi_{j^{\prime}}^{\prime}(H)\right|
$$

In essence, the roughness correlation function $\zeta(\mathbf{q})$ plays the same role for scattering by surface roughness as the impurity cross-section for scattering by static bulk impurities. It is known that surface scattering depends not only on the main parameters of roughness, such as its average amplitude or correlation radius, but also on the functional form of the correlation function (see, e.g., $[29,30]$ and references therein). Therefore, the proper identification of the surface correlator is paramount for the use of rough mirrors in precision measurements. It turns out that such accurate identification of the correlation function, which is necessary for precision experiments, is not trivial. The difficulties in identifying the correlation parameters go well beyond the recognized ones $[31,32]$ such as a tip profile [33] or the step size [34].

The purpose of this paper is to analyze and compare the properties of real and computationally generated rough mirrors and find out the limits on their use as state selectors by evaluating the accuracy of predictions for the observables. We also propose a radically new design for rough mirrors, what we call an Ising mirror, which might provide a much more controllable environment for experiment.

\section{Experiment}

2.1. Mirror Design and Roughness Measurements. The experimental part of the paper deals with a new large rough glass mirror produced for ongoing GRANIT experiments at ILL (Figure 1) [22-24].

The design and construction of the mirror are determined by the following considerations. This mirror should serve for shaping of the initial UCN spectrum and will be installed at the most upstream part of the transport mirror. In first test experiments, it will be used also for measurements of parameters of gravitational quantum states of UCNs. Since the principle application of the mirror is to serve as a gravitational state selector, our choice is to have large roughness amplitudes thus maximizing its efficiency at the

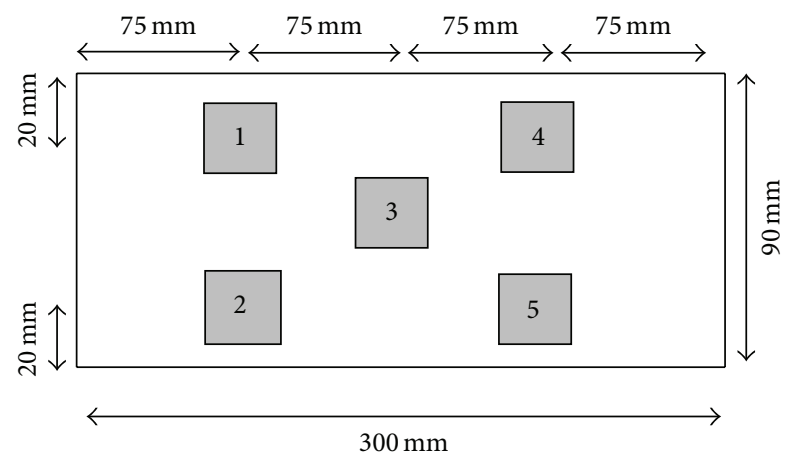

FIGURE 1: The rough mirror with five control patches. The surface roughness is assumed to be uniform across the mirror.

expense of precision of absolute measurements with this rough scatterer/absorber. The total length along the beam of UCNs is restricted by a length of the flat bottom mirror; therefore, we limited the length of the rough mirror by some reasonable small value $(9 \mathrm{~cm})$. The width of the rough mirror is similar to the width of the bottom mirror $(30 \mathrm{~cm})$.

The surface profile for this new mirror has been measured at ESRF using the vertical scanning interferometry (VSI) technique (see, e.g., [35]). Light is split into two coherent light beams. One beam is sent onto the measured mirror and is coupled after reflection with another light beam reflected from a perfectly flat sample (with the roughness of $0.5 \AA$ ). The resulting beam is analyzed in a CCD camera and the interference patterns give the profile of the rough mirror. Scanning over a certain surface area of the rough mirror allows one to measure the distribution of roughness. If the roughness is too sharp in a particular point, the beam deviates from its vertical trajectory and does not get back into the detector resulting in a "bad" point in the data set. The fabrication of the rough mirror favors producing moderate roughness without large angles between the local surface and the global reference plane (too large amplitudes have been eliminated by the surface production technique). There are about 1.5-2\% of "bad" points among the whole data. Most of the "bad" points are isolated, but some form small clusters (the largest cluster contains 8 "bad" points).

An alternative method to the VSI technique would have been the atomic force spectroscopy (AFM). However, using the VSI technique in this particular case had several advantages. First, the characteristic size of the surface to be scanned has to be much larger than the correlation length of the roughness, that is, at least a few hundreds of micrometers. Such areas are too large for a standard AFM. Second, the amplitude of the roughness should be comparable to the characteristic quantum gravitational length scale [2-6] equal to

$$
l_{0}=\hbar^{2 / 3}\left(2 m^{2} g\right)^{-1 / 3} \sim 5.871 \mu \mathrm{m}
$$

in order to provide high efficiency of neutron absorption in this rough mirror. Such amplitudes are too large for an AFM which measures irregularities in the angstrom range. 
The raw data set contains surface profiles $y_{i}(s, t)$ measured in five patches $(i=1 ; 2 ; 3 ; 4 ; 5)$, Figure 1 . The mirror is $90 \mathrm{~mm}$ long in the direction of the beam and $300 \mathrm{~mm}$ wide. The size of each patch is $0.504 \times 0.504 \mathrm{~mm}^{2}$. The data set for each patch contains $2,557 \times 2,557 \approx 6.5 \times 10^{6}$ points. The distance between individual data points is $0.19 \mu \mathrm{m}$ which is much smaller than $l_{0}$ and the characteristic amplitude and correlation length of the roughness.

2.2. Extraction of the Roughness Correlation Function. The data analysis consists of several steps. First, the "bad" points have been replaced by the averages of surrounding points. Second, the proper reference planes for individual patches are restored by fitting each of the five data sets $y_{i}(s, t)$ to the planes $a_{i}+b_{i} s+c_{i} t$ to eliminate the tilting and ensure the zero average for the roughness profile $\left\langle y_{i}(s, t)\right\rangle=0$. The set of coefficients $a_{i}, b_{i}, c_{i}$ provides the best fit to the profile data for the patch $i$ and the values of $a_{i}+b_{i} s+c_{i} t$ are subtracted from $y_{i}(s, t)$. By itself, eliminating the tilting in this way is not controversial. The only potential problem here could arise if the mirror as a whole is slightly curved and the reference planes for the individual patches are different from each other.

Since the primary designation of this mirror is to serve as a state selector in new GRANIT experiments aimed at selecting and identifying neutron states in quantizing gravitational field, it is convenient to measure all length parameters, including the roughness correlation function, in units of $l_{0}$, (3), which is the size of the lowest quantum state for a neutron in the Earth gravity field in open geometry. Below all lengths in the figures and tables are given in the corresponding dimensionless units.

The analysis of the data sets and the results are similar to [36] in which we analyze computationally generated rough surfaces. The two-dimensional (2D) correlation function is defined as

$$
\begin{aligned}
\zeta(s, t) & =\left\langle y\left(s^{\prime}, t^{\prime}\right) y\left(s^{\prime}+s, t^{\prime}+t\right)\right\rangle_{x, y} \\
& \equiv \frac{1}{A} \int y\left(s^{\prime}, t^{\prime}\right) y\left(s^{\prime}+s, t^{\prime}+t\right) d s^{\prime} d t^{\prime},
\end{aligned}
$$

where $A$ is the averaging area. The discrete analog of this equation is

$$
\zeta(s>0, t>0)=\frac{1}{(N-s)(N-t)} \sum_{t^{\prime}=1+t}^{N} \sum_{s^{\prime}=1}^{N-s} y_{t^{\prime}, s^{\prime}} y_{t^{\prime}-t, s^{\prime}+s^{\prime}} .
$$

We try to improve the accuracy by maximizing utilization of the data points though the finite size of the patches degrades the accuracy of the computations in (5) for large $s, t$. This degradation (the so-called wagging tails) becomes very rapid at $s, t>N / 2$.

The extraction of the correlation information from the profile data sets is routine with the only limitation being the large volume of profile data. A typical 2D correlation function for one of the patches is given in Figure 2 (light blue surface). For comparison, the dark blue surface presents the best fit of this correlation surface $\zeta(s, t)$ by an isotropic exponential

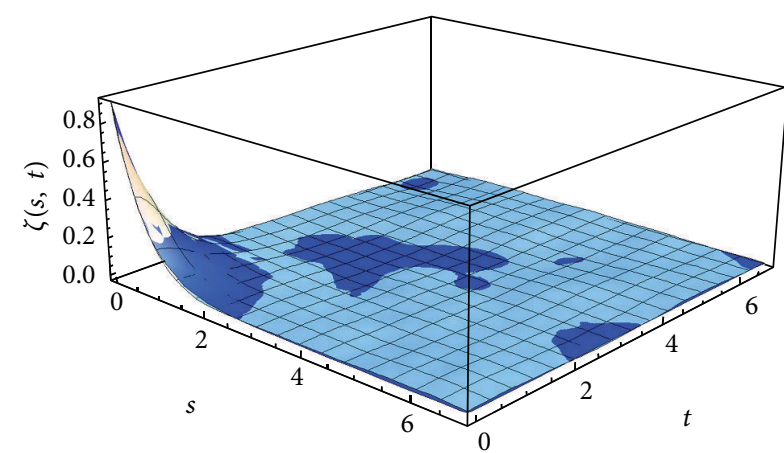

Figure 2: 2D correlation function $\zeta(s, t)$ for the Patch 5 (light blue surface). All axes are in units of $l_{0}$, (3). The darker surface is given by the best fit for $\zeta(s, t)$ by an isotropic exponential fitting function $\zeta(r)=\zeta(0,0) \exp \left(-r / r_{E}\right)$ with $r=\sqrt{s^{2}+t^{2}}$. The best fitting values of the correlation radius $r^{E}$ are summarized in Tables 1 and 2 for all five patches.

TABLE 1: The parameterization of the extracted correlation functions $\zeta(s, t)$ by anisotropic exponential and power law fitting functions, (6). Rows $1-5$ give the data for the individual patches and the last row for the correlation function averaged over all patches.

\begin{tabular}{lccc}
\hline$\#$ & $\eta$ & $r_{s}^{E}, r_{t}^{E}, \sigma_{E} \times 10^{2}$ & $r_{s}^{\mathrm{PL}}, r_{t}^{\mathrm{PL}}, u, \sigma_{\mathrm{PL}} \times 10^{2}$ \\
\hline 1 & 1.03 & $0.62,0.63,1.42$ & $0.45,0.45,0.9997,1.56$ \\
2 & 1.14 & $0.70,0.73,2.69$ & $0.44,0.46,0.864,2.04$ \\
3 & 0.97 & $0.64,0.74,2.71$ & $0.28,0.31,0.658,1.37$ \\
4 & 0.99 & $0.64,0.66,2.35$ & $0.32,0.34,0.73,1.26$ \\
5 & 0.96 & $0.57,0.60,0.83$ & $0.46,0.48,1.10,1.20$ \\
\hline Av. & 1.02 & $0.64,0.67,1.84$ & $0.38,0.40,0.842,1.33$ \\
\hline
\end{tabular}

surface. The figure demonstrates that the anisotropy of the roughness correlation function is noticeable but not very large.

We checked the isotropy of five extracted correlation functions by fitting them to anisotropic exponential and power law fitting functions:

$$
\begin{aligned}
& \zeta_{E}=\eta_{E}^{2} \exp \left(-\sqrt{\left(\frac{s}{r_{s}^{E}}\right)^{2}-\left(\frac{t}{r_{t}^{E}}\right)^{2}}\right), \\
& \zeta_{\mathrm{PL}}=\frac{\eta_{\mathrm{PL}}^{2}}{\left(1+\left(s / r_{s}^{\mathrm{PL}}\right)^{2}+\left(t / r_{t}^{\mathrm{PL}}\right)^{2}\right)^{u}} .
\end{aligned}
$$

The best fit parameters are summarized in Table 1 .

The first five rows in the table provide the data for the individual patches, while the last row describes the correlation function averaged over all patches. One can make two conclusions. First, the statistical quality of the fit by each fitting function $\zeta_{\text {fit }}$, which is measured by $\sigma_{\text {fit }}$,

$$
\sigma_{\text {fit }}^{2}=\frac{\left\langle\left(\zeta-\zeta_{\text {fit }}\right)^{2}\right\rangle}{\zeta^{2}(0,0)}
$$

for each patch is roughly the same for both fitting functions $\zeta_{E}$ and $\zeta_{\mathrm{PL}}$. Second, the anisotropy of the correlation function 
TABLE 2: Results of the fit of the extracted correlation functions for five patches and for the averaged correlation function (last row) by the exponential $(E)$, Gaussian $(G)$, and power law (PL) fitting functions, (8)-(10). For each fitting function the table shows the correlation radii $r_{E, G, P L}$ and the quality of the fits $\sigma$, (7). The last column shows the calculated parameter $\Phi_{E, G, P L}$ [37] responsible for the exit neutron count.

\begin{tabular}{lccccc}
\hline$\#$ & $\eta$ & $r_{E}, \sigma_{E} \times 10^{2}$ & $r_{G}, \sigma_{G} \times 10^{2}$ & $r_{\mathrm{PL}}, \sigma_{\mathrm{PL}} \times 10^{2}$ & $\left(\Phi_{E}, \Phi_{G}, \Phi_{\mathrm{PL}}\right) \times 10^{-3}$ \\
\hline 1 & 1.03 & $0.62,1.42$ & $0.49,3.93$ & $0.66,2.04$ & $5.44,3.94,4.18$ \\
2 & 1.14 & $0.72,2.44$ & $0.56,5.75$ & $0.76,3.15$ & $6.23,4.52,4.78$ \\
3 & 0.97 & $0.68,2.61$ & $0.50,4.94$ & $0.70,3.23$ & $4.63,3.46,3.6$ \\
4 & 0.99 & $0.65,2.25$ & $0.49,4.56$ & $0.68,2.80$ & $4.92,3.64,3.81$ \\
5 & 0.96 & $0.58,0.68$ & $0.47,3.0$ & $0.62,1.48$ & $4.87,3.50,3.75$ \\
\hline Av. & 1.02 & $0.65,1.75$ & $0.50,4.39$ & $0.69,2.44$ & $5.22,3.83,4.01$ \\
\hline
\end{tabular}

as measured by difference in the correlation radii $r_{s, t}$ in $s$ and $t$ directions is of the order of several percent and is small, smaller than the difference between the patches which is rather pronounced. For this reason we neglect the anisotropy and concentrate on analyzing the extracted correlation functions averaged over the angles, $\zeta(x), x=$ $\sqrt{s^{2}+t^{2}}$.

2.3. Identification of the Correlation Function. We fitted the correlation functions averaged over the angles with the isotropic exponential, Gaussian, and power law fitting functions:

$$
\begin{gathered}
\zeta_{E}=\eta^{2} \exp \left(\frac{-x}{r_{E}}\right), \\
\zeta_{G}=\eta^{2} \exp \left(\frac{-x^{2}}{2 r_{G}^{2}}\right), \\
\zeta_{\mathrm{PL}}=\frac{\eta^{2}}{\left(1+x^{2} / r_{\mathrm{PL}}^{2}\right)^{3 / 2}} .
\end{gathered}
$$

The results of best fits are summarized in Table 2 .

Table 2 is structured similarly to Table 1 but has an extra column with $\Phi_{E, G \text {,PL }}$ calculated using the fitting functions with parameters from the table. Without going into details, we just mention that $\Phi$ is a normalized linewidth for neutrons in the lowest gravitational state and is a complicated integral of the power spectrum $\zeta(\mathbf{q})$. Its value strongly depends not only on the time of flight through the waveguide, dimensionality of roughness, correlation radius, and amplitude of inhomogeneities, but also on the functional form of $\zeta(\mathbf{q})$ [37]. The value of $\Phi$ serves here as a descriptor for observables: it determines the exit neutron count for GRANIT-type experiments (see Section 4). In essence, the appropriateness of the choice of the fitting functions should be judged not by the standard deviation $\sigma$ but by the value of $\Phi$. But which value of $\Phi$ from the table is the closest to the "true" value of $\Phi$ in experiments with this mirror?

The statistical quality of the fits $\sigma$ for all three fitting functions are close to each other, while the values of the observables, in this case $\Phi$, are noticeably different. An explanation is simple. A typical correlation function consists of a peak area and long tail. For finite size samples the fluctuation-driven tail is rather fat and does not go to zero at large distances. As a result, the standard deviation $\sigma$ between the extracted correlator and any reasonable fitting function, which goes to zero relatively fast at large distances, is determined not by the peak area where the fitting functions differ from each other, but by the contributions from the tails, where all the fitting functions are zero. As a result, the values of $\sigma$ for all three fitting functions are similar, while the values of the observable, $\Phi$, are noticeably different. The main conclusion is that selection of the fitting function could not and should not be judged just by the value of $\sigma$, (7). An increase in sample size does not help much: though the sizedriven fluctuations and, therefore, $\sigma$ decrease with increasing sample as $\sqrt{2 / N}$ ( $N$ is the sample size), the tails get longer with increasing $N$, and the values of $\sigma$ go down for all fitting functions in the same way. We will explore this point in more detail in the next section.

There are several ways how to suppress fluctuations and make identification of the correlation function easier. One can average the correlation function over several samples and use the averaged values for identification as in, for example, $[38,39]$. This is done in the last row in Tables 1 and 2 . This assumes that the correlations are the same in all samples and that any long-range oscillations are non-physical and are purely fluctuation-driven. One can also disregard the tails and use for identification purposes only the peak and its immediate vicinity thus ignoring the long-range tails as spurious. We need to develop a reliable identification procedure. Our recommendation, based on the results of the next section, is given in the beginning of Section 4 .

\section{Numerical Experiments}

The difficulties that we face when trying to identify the roughness correlation function for the rough mirror are typical. The best way to overcome these difficulties would be extracting the correlation functions from profile measurements for surfaces with known roughness correlation functions and comparing the results with these known correlators. Unfortunately, it is virtually impossible to prepare the surfaces with the predetermined roughness correlation functions.

The best available alternative is to numerically generate surfaces with the predetermined roughness correlators and extract the correlation function from a numerical experiment which emulates precise scanning measurements of the surface profile. Below we report numerical experiments of this 


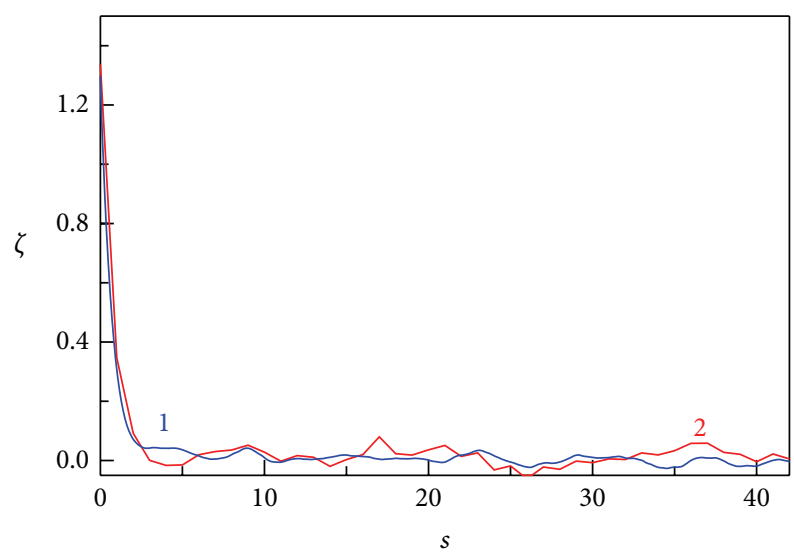

FIgURE 3: The illustration of the fluctuation-driven tails of the correlation function $\zeta(s, 0)$ for the Patch 2 of the actual mirror (curve 1, blue) and for the numerically generated surface with roughness emulating the exponential correlation function with the same amplitude and the correlation radius as in Table 2 (curve 2, red).

type and compare the extracted information with the physical experiments on the rough mirror in Section 2. The results allowed us to better understand the accuracy and pitfalls of the identification procedures.

\subsection{Surfaces with any Predetermined Roughness Correlators.} We can generate surfaces with any predetermined discrete correlation function $\widehat{\zeta}_{i k}=\zeta(i-k)$. The technique starts from using the Gaussian distribution, which is embedded in most random number generators, for generating uncorrelated random numbers $g_{i}$ with the $\delta$-type correlation function (white noise), $\left\langle g_{i} g_{k}\right\rangle=\delta_{i k}$. We then rotate this vector $\vec{g}$ using the rotation matrix $\widehat{A}=\widehat{\zeta}^{1 / 2}$. The resulting set of numbers $y_{i}, \vec{y}=\widehat{A} \vec{g}$, exhibits desired correlations, $\left\langle y_{i} y_{k}\right\rangle=\zeta_{i k}$ (for more details see $[36,37])$. Next we "measure" the generated profile $y_{i}=y\left(x_{i}\right)$ by emulating the scanning technique and extract the "experimental" correlation function. We then try to identify this extracted correlator by fitting it to various fitting functions (in practice, Gaussian, exponential, and power law functions), calculate the observables (for the purpose of this paper, $\Phi$ ), and compare the results with those obtained using the "true" correlation function $\zeta_{i k}$. Below we report such experiments for both $1 \mathrm{D}$ and $2 \mathrm{D}$ roughness. For computational reasons the linear sizes of our 2D samples are smaller than in $1 \mathrm{D}$ cases, though the overall number of profile points is larger.

The difficulties that we encounter in our numerical experiments are exactly the same as in our analysis of the real mirror in Section 2. The main source of uncertainty is the fluctuations related to the final size of the samples. For illustration, in Figure 3 we plot the fluctuation-driven tails for the extracted roughness correlation function $\zeta(s, 0)$ for Patch 2 of the actual mirror (curve 1, blue) and for the correlation function $\zeta(x)$ for a generated 1D rough surface of the same size $(2,500$ points; curve 2 , red) which emulates roughness with the exponential correlation function $\zeta=\eta^{2} \exp (-x / r)$ with $\eta=1.14$ and $r=0.72$ as for this patch in Table 2 . The similarity between these two fluctuation-driven tails is striking.

Our ability to generate surfaces with roughness emulating any predetermined correlation function allows us to analyze the use of fitting functions for our extracted correlators and to determine whether it is possible to avoid using the fitting functions by inputting the raw correlation data directly into equations for observables $(\Phi)$. Some of our findings are summarized in the following two tables.

The quality of the fits $\sigma_{G}, \sigma_{E}, \sigma_{\mathrm{PL}}$ for all three types of the fitting functions is more or less the same, about $5 \times$ $10^{-4}$, but the results for the physically important parameter $\Phi_{1 G, E, \mathrm{PL}}$, which predicts the exit neutron count in GRANIT experiments, differ considerably from each other by about $25 \%$. In our numerical experiment, the "true" shape of the correlation function is known to be Gaussian and, not surprisingly, the fitting by the Gaussian function yields the values of $\Phi_{1}$ very close to the "true" value 23.48 . This brings us to an inevitable conclusion that the statistical quality of the fit $\sigma$ by any reasonable ad hoc fitting function $\zeta_{\text {fit }}$, (7), does not predict the quality of physical conclusions obtained using this fitting function. Note that the results for fitting by the power law and exponential correlation functions are relatively close to each other and very different from those for the Gaussian fit. The explanation is simple: the Gaussian function has a much shorter tail.

The last column in Table 3 shows the values of $\Phi_{1 n}$ which are calculated by inputting the discrete raw correlation data directly into the equations for $\Phi_{1}$. In $1 \mathrm{D}$ this procedure works better than using the fitting function of the "wrong" shapes $\zeta_{E, \text { PL }}$, but still noticeably worse than for the "right" fitting function $\zeta_{G}$ though the statistical quality $\sigma_{n}$ of the used spectral decomposition of the raw data $\sigma_{n}$ is perfect.

The 2D results (Table 4 ) are different because of different dimensionality and smaller linear sizes of our samples. Here as an observable, which is used to compare the results, we use $\Phi_{2}$ which describes the neutron count in experiments with $2 \mathrm{D}$ roughness $[38,41]$. The generated rough surfaces are emulating the Gaussian roughness with the correlation function $\zeta(|\mathbf{x}|)=\exp \left(-|\mathbf{x}|^{2} / 8\right)$ (i.e., $\eta=1, r=2$ ) for which $\Phi_{2}=2.58 \times 10^{3}$. The sample size is $61 \times 61$ points. The table contains the results extracted from the best fit of the extracted correlator to the Gaussian, exponential, and power law functions. The Gaussian fit is done independently for the correlation function $\zeta(|\mathbf{x}|)$ averaged over the angles and the $2 \mathrm{D}$ correlation function $\zeta(\mathbf{x})$; the results are close to each other. The statistical quality of the fits $\sigma$ is worse than in the $1 \mathrm{D}$ case though the overall number of the data points in our $2 \mathrm{D}$ computations is larger (3,600 versus 2,000 points): the linear size of the sample is noticeably smaller while the correlation radius is slightly bigger. Table 4 provides the values of the extracted fitting parameters $\eta$ and $r$, values of $\sigma$, and, most importantly, the corresponding values of the physical observable $\Phi_{2}$. The results for direct use of numerical data without fitting are too unstable to be included in the table; we are not sure whether this is a result of higher dimensionality or smaller linear sizes of the samples. The 
TABLE 3: Three numerical runs for 1D surfaces emulate the Gaussian correlation of inhomogeneities $\eta^{2} \exp \left(-x^{2} / 2 r^{2}\right)$ with $r=1.19$ and $\eta=0.119$ (the assumed [37] roughness of the mirror in earlier GRANIT experiments [2-4,6]). The true value of the observable $\Phi_{1}$ for such a surface is $\Phi_{1}=23.48$. The extracted correlators are fitted with Gaussian, $\eta_{G} \exp \left(-x^{2} / 2 r_{G}\right)$, exponential, $\eta_{E} \exp \left(-x / r_{E}\right)$, and power law, $\eta_{\mathrm{PL}} /\left[1+\left(x / r_{\mathrm{PL}}\right)^{2}\right]^{3 / 2}$ fitting functions. The table contains the best fitting values of $r_{G, E, \mathrm{PL}}$, statistical quality of the fits $\sigma_{G, E, \mathrm{PL}}$, and the recalculated values of $\Phi_{1 G, E, P L}$. The best fitting values of $\eta_{G, E, P L}$ are close to each other and are not listed. The columns with $\Phi_{n}$ and $\sigma_{n}$ give the values of $\Phi_{1}$ and the standard deviation when the spectral decomposition of the raw correlation data is put directly into equations for $\Phi_{1}$ [37] without using the fitting functions.

\begin{tabular}{cccccc}
\hline$\#$ & $r_{G}, \sigma_{G} \times 10^{4}$ & $r_{E}, \sigma_{E} \times 10^{4}$ & $r_{\mathrm{PL}}, \sigma_{\mathrm{PL}} \times 10^{4}$ & $\sigma_{n} \times 10^{17}$ & $\Phi_{1 G}, \Phi_{1 E}, \Phi_{1 \mathrm{PL}}, \Phi_{1 n}$ \\
\hline 1 & $1.19,5.24$ & $1.59,5.81$ & $1.44,5.81$ & 1.92 & $23.86,18.19,18.81,21.96$ \\
2 & $1.15,4.49$ & $1.53,4.56$ & $1.36,4.64$ & 1.83 & $23.33,17.84,18.65,21.14$ \\
3 & $1.25,4.37$ & $1.69,4.40$ & $1.54,4.47$ & 1.69 & $23.56,17.26,17.85,20.96$ \\
\hline
\end{tabular}

TABle 4: The same as in Table 3 for generated 2D rough Gaussian surfaces with $r=2$ and $\eta=1$. The expected value of $\Phi_{2} \approx 2.58 \times 10^{3}$. The table contains the extracted fitting parameters $\eta_{G, E, \text { PL }}^{\text {fit }}$ and $r_{G, E, \text { PL }}^{\text {fit }}$, together with $\sigma_{G, E, \text { PL }}$, and the recalculated values of $\Phi_{2}$. The fourth row gives the results for the correlation function averaged over 10 independent runs.

\begin{tabular}{lcccc}
\hline$\#$ & $\eta_{G}, r_{G}, \sigma \times 10^{2}$ & $\eta_{E}, r_{E}, \sigma \times 10^{2}$ & $\eta_{\mathrm{PL}}, r_{\mathrm{PL}}, \zeta_{0}^{\mathrm{PL}}, \sigma \times 10^{2}$ & $\left(\Phi_{2}^{G}, \Phi_{2}^{E}, \Phi_{2}^{\mathrm{PL}}\right) \times 10^{-3}$ \\
\hline 1 & $1.04,1.97,5.7$ & $1.14,2.04,6.2$ & $1.08,2.45,5.9$ & $2.81,5.51,1.68$ \\
2 & $1.10,1.80,6.5$ & $1.20,1.76,7.4$ & $1.14,2.15,7.2$ & $3.23,6.56,1.99$ \\
3 & $0.90,1.84,4.1$ & $0.98,2.05,4.3$ & $0.94,2.40,4.1$ & $2.18,4.06,1.28$ \\
\hline Av. & $1.00,1.98,1.9$ & $1.10,2.11,2.9$ & $1.05,2.49,2.4$ & $2.60,5.05,1.57$ \\
\hline
\end{tabular}

table contains results of three numerical runs and (the fourth row) the average for ten numerical runs.

\subsection{Surfaces with Quantized Amplitudes of Inhomogeneities:} Ising Roughness. The above approach allow us to create and analyze random rough surfaces with arbitrary correlation functions. The drawback of our procedure is that while it is appropriate for macroscopic roughness, it cannot produce roughness with quantized amplitudes which is desirable for the study of atomic-scale roughness.

It might be impossible to computationally emulate a random rough surface with an integer profile $y\left(x_{i}\right)$ with an arbitrary predetermined correlation function $\widehat{\zeta}(i-k)=$ $\left\langle y_{i} y_{k}\right\rangle$ except, of course, for "classical" surfaces with very large amplitude of roughness. However, several specific "quantized" correlators can still be generated using Monte Carlo simulations for spin lattice models with various Hamiltonians. This might help in extracting the proper correlation functions from experimental data on the surface profile based on realistic assumptions on the interaction of the surface defects. This can also help to guess which correlation functions to use in theoretical calculations. Needless to say, many of the lattice models produce the correlation functions which are exponential at large distances and have complicated, often analytically unresolved structure in the peak area.

Unfortunately, the universe of the correlation functions which are accessible in this way is limited by the number of known exactly solvable lattice models, mostly in $1 \mathrm{D}$, some of which may have little resemblance to real surfaces. It is even unclear whether there are any restrictions on allowed forms of the correlation functions. In 2D even the simplest models, such as the Ising model, lead to the correlation functions for which we do not have explicit analytical expressions making them virtually useless for our purposes.
The simplest example [36] is, of course, the ferromagnetic Ising lattice $y_{i}= \pm 1$ for which the correlation function is determined by the attractive coupling constant $J$ in the Hamiltonian (or, what is the same, by the Boltzmann factors $\exp ( \pm 2 J / k T))$. In the $1 \mathrm{D}$ case the correlation function is exponential:

$$
\zeta_{E}(x)=\eta^{2} \exp \left(-\frac{x}{r}\right), \quad r=\frac{1}{2} \exp \left(\frac{2 J}{k T}\right) .
$$

The correlation function for the $2 \mathrm{D}$ Ising model, though known in principle, [40, 42], is described by a set of complicated equations involving elliptical integrals.

In $1 \mathrm{D}$ computations we used 1000 positions $x_{i}$ and performed $10^{6}$ Monte Carlo cycles. The correlation function $\zeta(s)$ should emulate function (11) with $r=R / l_{0}=1.19$ and $\eta=\ell / l_{0}=0.119$ as in the earlier GRANIT experiments. The results for five runs are summarized in Table 5 . The true value of $\Phi_{1}$ for the exponential correlation function with $r=1.19$ and $\eta=0.119$ is $\Phi_{E}^{\text {th }}=19.5$ (with the same values of $r$ and $\eta, \Phi_{G}^{\text {th }}=23.7$ and $\left.\Phi_{\mathrm{PL}}^{\text {th }}=20.4\right)$. Since the simulation is based on the Ising model with spins \pm 1 , the extracted average amplitudes of roughness differ from $\eta=0.119$ by less than $1 \%$ for all fitting functions and there is no need to present the values of $\eta_{E, G, P L}$. Of course, the fit using the exponential correlator provides the best values for $\Phi_{1}$ though here again the values of $\sigma$ for all fitting functions are practically indistinguishable. Of the other two fits, it is not clear why the power law fit provides much better values of $\Phi_{1}$ than the Gaussian one. The last column in the table also shows the values of $\Phi_{n}$ which are obtained by direct spectral analysis with $N / 2$ harmonics of the raw correlation data without any fitting. These data display the worst agreement with $\Phi_{E}^{\text {th }}=$ 19.5 , while the value of $\sigma_{n}$ is by 13 orders of magnitude better than $\sigma$ for any of our fitting functions. The explanation is the 
TABLE 5: Five Monte Carlo runs for the 1D Ising model. The "true" correlation function is exponential with $r=1.19$ and $\eta=0.119$ and yields $\Phi_{E}^{\text {th }}=1$ 19.5. The correlation functions extracted from the generated rough surfaces were fitted with the exponential, Gaussian, and power law functions. The Table contains the best fitting values of $r_{E, G, P L}$ and the corresponding values of $\sigma_{E, G, P L}$ and $\Phi_{E, G, P L}$. Since the simulation is based on the Ising model with spins \pm 1 , the best fitting values of $\eta$ differed from 0.119 by less than $1 \%$ for all fitting functions. The values of $\Phi_{n}$ were obtained by direct spectral decomposition of the raw correlation data. The size of the sample was $N=1000$ and we performed $10^{6}$ Metropolis cycles.

\begin{tabular}{cccccr}
\hline$\#$ & $r_{E}, \sigma_{E} \times 10^{4}$ & $r_{G}, \sigma_{G} \times 10^{4}$ & $r_{\mathrm{PL}}, \sigma_{\mathrm{PL}} \times 10^{4}$ & $\sigma_{n} \times 10^{17}$ & $\Phi_{1}^{E}, \Phi_{1}^{G}, \Phi_{1}^{\mathrm{PL}}, \Phi_{n}$ \\
\hline 1 & $1.27,6.69$ & $0.85,6.93$ & $1.26,6.72$ & 3.79 & $18.6,27.4,19.6,25.8$ \\
2 & $1.23,6.83$ & $0.88,6.94$ & $1.25,6.84$ & 1.49 & $19.1,26.8,19.7,26.2$ \\
3 & $1.04,6.51$ & $0.73,6.74$ & $1.07,6.54$ & 2.82 & $20.7,30.2,21.4,27.3$ \\
4 & $1.18,6.65$ & $0.87,6.71$ & $1.23,6.62$ & 3.01 & $19.7,27.1,20.0,26.1$ \\
5 & $0.94,6.44$ & $0.74,6.42$ & $1.03,6.38$ & 1.91 & $22.2,29.8,21.9,27.7$ \\
\hline
\end{tabular}

TABLE 6: Results for three rough surfaces generated using the 2D Ising model (the first three rows) and for the correlation function averaged over ten runs (the last row). The Monte Carlo simulations have been done at $T=1.2 T_{c}$ with $10^{6}$ Metropolis cycles. The surface size is $101 \times 101$. The Table is arranged similarly to Table 2 . The table contains the best fitting values of $r_{E, G, \mathrm{PL}}$ and the corresponding values of $\sigma_{E, G, \mathrm{PL}}$ and $\Phi_{2}^{E, G, \mathrm{PL}}$. The results for the exponential fits $\Phi_{2}^{E 1,2}$ for $\zeta(|\mathbf{x}|)$ and $\zeta(\mathbf{x})$ should be the closest to the true physical parameters.

\begin{tabular}{lccccc}
\hline$\#$ & $r_{E 1}, \sigma_{E 1} \times 10^{2}$ & $r_{E 2}, \sigma_{E 2} \times 10^{2}$ & $r_{G}, \sigma_{G} \times 10^{2}$ & $r_{\mathrm{PL}}, \sigma_{\mathrm{PL}} \times 10^{2}$ & $\left(\Phi_{2}^{E 1}, \Phi_{2}^{E 2}, \Phi_{2}^{G}, \Phi_{2}^{\mathrm{PL}}\right) \times 10^{3}$ \\
\hline 1 & $1.56,2.03$ & $1.60,2.75$ & $1.06,2.41$ & $1.55,2.12$ & $3.37,3.32,2.53,2.58$ \\
2 & $1.43,1.56$ & $1.43,2.27$ & $1.06,1.89$ & $1.48,1.63$ & $2.53,2.53,2.53,2.64$ \\
3 & $1.53,1.66$ & $1.53,2.49$ & $1.11,2.04$ & $1.57,1.75$ & $3.40,3.40,2.48,2.57$ \\
\hline Av. & $1.54,0.69$ & $1.57,0.89$ & $1.10,1.42$ & $1.57,0.91$ & $3.39,3.36,2.49,2.57$ \\
\hline
\end{tabular}

same as before: the full set of raw data is dominated by the long correlation tails which come from the fluctuations.

The last table, Table 6 , presents results for three rough surfaces generated using the $2 \mathrm{D}$ Ising model plus a row for the correlation function averaged over ten runs. The observable here is again $\Phi_{2}$.

The computations are done above the phase transition, $T=1.2 T_{c}$. At this temperature the correlation function is, probably, still close to the exponential, but it is not clear how close. Here we do not know exactly what should be the "true" value of $\Phi_{2}$ but expect that the exponential correlator provides the best estimate. At this temperature the domains are relatively small and the relaxation times are manageable. The size of the surface is relatively large, $101 \times 101$, and each computation runs $10^{6}$ Metropolis cycles. The table is arranged similarly to Table 5 . The values of $\sigma$ for all fitting functions are again close to each other, while the values of $\Phi_{2}$ and $r$ are noticeably different. The results for the exponential fit should be the closest to the true physical parameters. The first column for the exponential fitting gives results obtained from the flat file $\zeta(|\mathbf{s}|)$. The second column gives the results of fitting $\zeta(\mathbf{s})$ by the $2 \mathrm{D}$ exponential function. For the Gaussian and power law correlators, columns 3 and 4, we used only the flat files $\zeta(|\mathbf{s}|)$. What is somewhat surprising is that the results for our choice of the power law correlator, which is the Fourier image of the exponential one, are again close to those using the exponential fit. What is even more surprising, the values of $\Phi_{2}$ for the power law fit using $\zeta(|\mathbf{s}|)$ are systematically closer to the exponential fit using $2 \mathrm{D} \zeta(\mathbf{s})$ than to the exponential fit using $\zeta(|\mathbf{s}|)$. The Gaussian fit yields very different $\Phi_{2}$, while the value of $\sigma$ is comparable with the others. The direct spectral analysis of the raw correlator data again yields the worst physical results and changes from run to run; these results are not even worth listing. The spectral analysis of the correlation function averaged over ten runs worked slightly better than the Gaussian fit. The difference between results obtained using different fitting functions once again illustrates the uncertainty in comparing computational and experimental data to theoretical results. One should have at least some information about the shape of the "true" correlation function.

\section{Experimental Consequences}

The main conclusion from the previous sections is that the difficulties that we experience trying to identify the correlation function for the actual mirror are exactly the same as in our numerical experiments. Our extensive numerical data show that the best way of identification seems to be the averaging over several samples in combination with the graphical and numerical analysis of the peak area without much reliance on the overall standard deviation $\sigma$. We do not recommend putting the discrete raw correlation data directly into equations for the observables.

Our reluctant conclusion from the analysis of experimental data on the roughness of the actual mirror in Section 2 is that its roughness correlation function is close to the isotropic exponential function (8) with the amplitude $\eta=1.02$ and the correlation radius $r=0.65$. This yields the value of $\Phi_{2} \approx 5.22 \times 10^{3}$. The uncertainty in parameters is about $10 \%$ because of the presence of bad points in the data sets, residual anisotropy, and, most of all, difference in values between the patches.

Knowing the value of $\Phi_{2}$ we can predict the exit neutron count in experiment with this mirror. Luckily, the uncertainty in $\Phi_{2}$ in this range of values, in contrast to smaller $\Phi$ in 


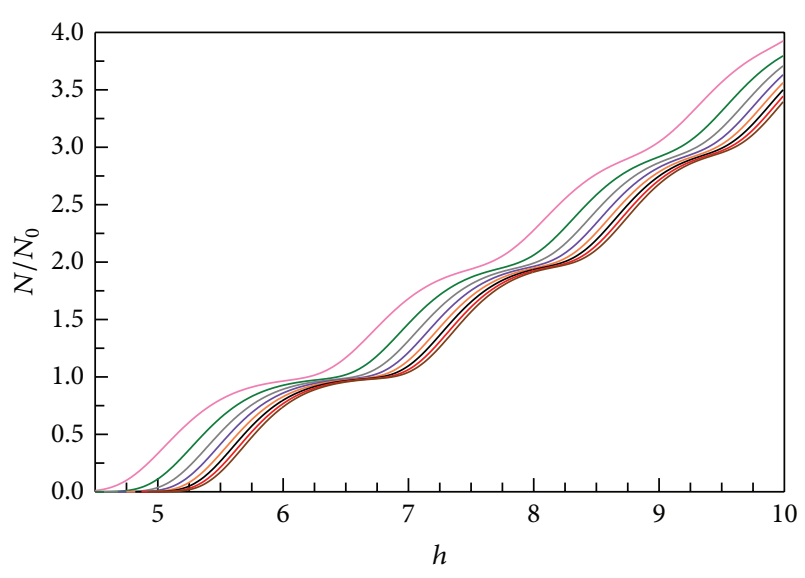

FIgURE 4: The exit neutron count $N_{e} / N_{0}$ as a function of the slit width $h$ for several values of $\Phi_{2}$ close to $5 \times 10^{3}\left(N_{0}\right.$ is the number of neutrons entering the slit in each quantum state). Eight curves from left to right correspond to $\Phi_{2} \times 10^{-3}=1 ; 2 ; 3 ; 4 ; 5 ; 6 ; 7 ; 8$; neutron count decreases with increasing $\Phi_{2}$.

$[37,43,44]$, does not affect the dependence of the exit neutron count on the slit width much (see Figure 4). Eight curves in the figure correspond to the dependence of the exit neutron count $N / N_{0}$ on the slit width $h$ for $\Phi_{2} \times 10^{-3}=$ $1 ; 2 ; 3 ; 4 ; 5 ; 6 ; 7 ; 8\left(N_{0}\right.$ is the number of neutrons in each state entering the slit). All curves in Figure 4 demonstrate the well pronounced predicted quantum steps which is a very good news for the ongoing experiment.

There are factors that might limit the accuracy of this prediction. On the theoretical side, the value of the average amplitude of roughness $\eta$ is too large considering the main theoretical assumption $\eta \ll r, h$. Also, there is still some uncertainty in the correlation parameters.

From the point of view of experiment, a large value of $\eta$ is also not very good. It results in a noticeable broadening of the levels and in inability to measure precisely the height of the reference plane and, therefore, the width of the slit. The latter factor leads to uncertainty in the values of quantized energy levels thus limiting the use of the setup for precision measurements of fundamental forces.

In addition, both theory and experiment require better information on the distribution of neutrons entering the slit over the quantum states.

\section{An Alternative Design: Ising Mirror}

Many of the sources of errors mentioned above disappear if one uses an alternative mirror design based on the 1D Ising model described in Section 3. In essence, we are proposing to make the rough mirror like an interferometric grating with trenches of constant depth but with randomly varying widths and separations [36]. Such an Ising grating can be easily generated computationally as it is done in Section 3 . Since the characteristic length is macroscopic, $l_{0}=\hbar^{2 / 3}\left(2 \mathrm{~m}^{2} \mathrm{~g}\right)^{-1 / 3} \sim$ $5.871 \mu \mathrm{m}$, the generated pattern can then be transferred to the mirror surface.

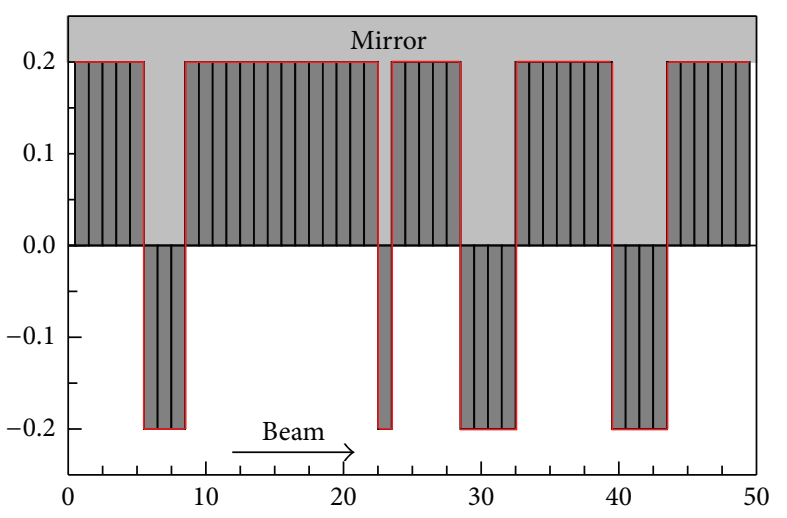

FIGURE 5: A fragment of the proposed rough upper mirror based on Monte Carlo simulations $\left(10^{6}\right.$ cycles) for the $1 \mathrm{D}$ Ising model with $J / k T \approx 0.7$. Thick red line is the surface profile and dark bars are the "Ising spins." Mirror material is above the red line (the filled area). The amplitude of roughness $\eta=0.2$, the correlation radius $r=2$, and the value of $\Phi_{1} \approx 43.5$. Both axes are in units of $l_{0}=\hbar^{2 / 3}\left(2 \mathrm{~m}^{2} \mathrm{~g}\right)^{-1 / 3} \sim 5.871 \mu \mathrm{m}$. The vertical scale is about 100 times smaller than the horizontal one. The value of $\eta$ can be changed simply by rescaling the vertical axis.

An element of such a mirror is plotted in Figure 5. The thick (red) line shows the surface of the mirror (since this is an upper mirror for a GRANIT-like experiment, the mirror material is above the red line and the neutron beam is propagating below it). The dark rectangles of the width $\ell_{0}$ are the "Ising spins." Both axes are measured in units of $\ell_{0}$, but the vertical scale is about 100 times larger than the horizontal one and the roughness is actually very mild.

Since the real mirror is continuous in contrast to the discrete Ising model, the roughness correlation function coincides with the Ising exponent (11) only in the integer points and is slightly different elsewhere:

$$
\begin{aligned}
\zeta(x)=\eta^{2}[ & \exp \left(-\frac{\lfloor x\rfloor}{r}\right)+(x-\lfloor x\rfloor) \\
& \left.\times\left(\exp \left(-\frac{\lceil x\rceil}{r}\right)-\exp \left(-\frac{\lfloor x\rfloor}{r}\right)\right)\right] .
\end{aligned}
$$

The neutron count predictor $\Phi_{1}$ for the correlation function (12) can be calculated only numerically. It differs from the one for a purely exponential function by not more than $5 \%$. The values of $\Phi_{1}$ with $r=2$ and $\eta=0.2 \div 0.4$ are in the $42.5 \div 170$ range with the original waveguide parameters and in the $30.3 \div 121.5$ range with the new ones. Because the amplitude of roughness is quite small, these values are much smaller than the ones in Figure 4 and the quantum steps are less pronounced (Figure 6).

Most of the factors, which are listed in the previous section as affecting the accuracy, disappear for this design. The width of the slit can be easily measured and the energy levels are much better defined. The design is easily scalable in both vertical and horizontal directions and creates a wellcontrolled environment. 


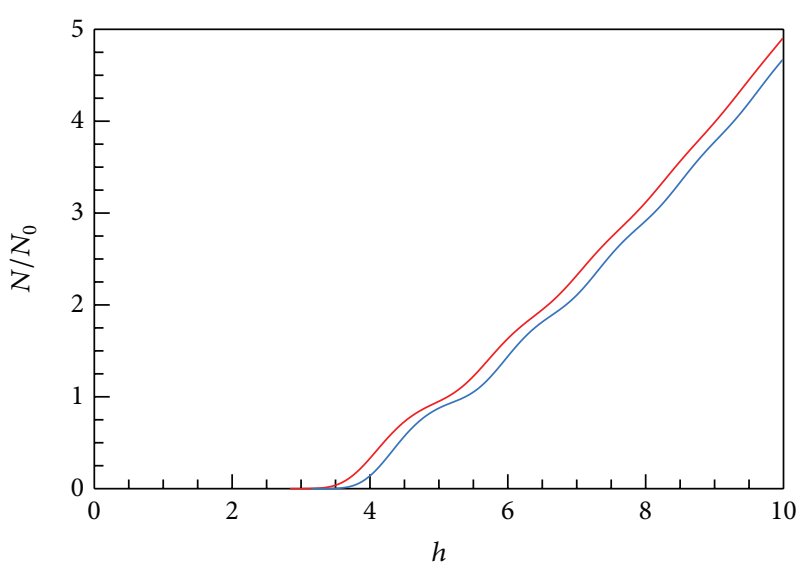

Figure 6: Predicted exit neutron count $N(h) / N_{0}$ for the mirror similar to the one in Figure 5 with roughness generated using Monte Carlo simulations for the 1D Ising model. The roughness amplitudes are $\eta=0.3$ (red) and $\eta=0.4$ (blue) and the correlation radius $r=2$. One can make steps more pronounced by further increasing $\eta$.

\section{Summary and Conclusions}

In summary, we analyzed roughness of the rough mirror built as a gravitational quantum state selector for a new cycle of GRANIT experiments. We extracted the correlation parameters of this mirror which are important for determining its scattering properties. The straightforward identification of the correlation function $\zeta(\mathbf{q})$ was impossible because of the unavoidable fluctuations associated with the finite sizes of the samples. All reasonable fitting functions $\zeta_{\text {fit }}$ exhibited more or less the same statistical quality of the fit $\sigma$, (7) but led to considerably different predictions for the observables.

Independently, we performed a series of numerical experiments aimed at identification of the correlation function extracted by the same techniques as used for the actual mirror from computationally generated rough surfaces with predetermined $\zeta(\mathbf{q})$. Remarkably, the difficulties in the identification of the correlation functions in our numerical experiments were the same as we experienced with the real rough mirror and were associated with similar fluctuationdriven fat tails. However, since in numerical experiments we knew the true correlation functions, we were able to hone our identification techniques.

In our numerical experiments we also tried avoiding the use of fitting functions by inputting the spectral decomposition of extracted raw correlation data directly into the equations for the observables. This option turned out to be marginally acceptable for $1 \mathrm{D}$ rough surfaces and did not work at all for 2D surfaces. Our main conclusion here is that the direct use of the raw correlation data is worse than the careful analysis of the fitting functions.

The results of our numerical experiments led us to believe that the proper identification of $\zeta(\mathbf{q})$ required, in addition to measuring the statistical quality of the fittings $\sigma$, averaging of the correlation functions extracted from several independent samples plus graphical and numerical analysis of the averaged correlator in the peak area. Based on this experience, we identified the correlation function for the actual mirror as an exponential one and obtained its parameters. This information allowed us to predict the exit neutron count for new GRANIT experiments utilizing this rough mirror as a function of the distance between the flat and rough mirrors. The predicted neutron count exhibited well-formed quantum steps which would allow us to use the setup for planned measurements of fundamental forces.

We also propose a radically new design for rough mirrors-what we called the Ising mirrors-for use as state selectors. The design is based on the Monte Carlo simulation of a $1 \mathrm{D}$ random pattern based on the $1 \mathrm{D}$ Ising model which is then transferred as a grating onto the actual mirror. Such transfer is feasible when the characteristic length is macroscopic as it is for the gravitational quantum state of neutrons in GRANIT experiments (about $6 \mu \mathrm{m}$ ). Such Ising mirror should look like an interference grating of straight 1D trenches of the same depths but with randomly varying widths and separations. Since the correlation function for such a random surface is known, one can easily get the reliable predictors for the observables which depend on scattering parameters. In addition, the environment is much better controllable than for usual rough mirrors for which the random variations in profile heights from point to point makes it extremely difficult, if not impossible, to determine the reference plane and accurately measure the height of the mirror.

\section{Conflict of Interests}

The authors declare that they have no conflict of interests regarding the publication of this paper.

\section{Acknowledgments}

The results of this paper were reported at GRANIT-2014 Workshop (Les Houches, France, March 2-7, 2014). One of the authors (A. E. Meyerovich) is grateful to the organizers and other members of GRANIT collaboration for the hospitality and support during the Workshop.

\section{References}

[1] V. V. Nesvizhevsky, H. Börner, A. M. Gagarski et al., "Search for quantum states of the neutron in a gravitational field: gravitational levels," Nuclear Instruments and Methods in Physics Research A: Accelerators, Spectrometers, Detectors and Associated Equipment, vol. 440, no. 3, pp. 754-759, 2000.

[2] V. V. Nesvizhevsky, H. G. Börner, A. K. Petukhov et al., "Quantum states of neutrons in the Earth's gravitational field," Nature, vol. 415, no. 6869, pp. 297-299, 2002.

[3] V. V. Nesvizhevsky, H. G. Börner, A. M. Gagarski et al., "Measurement of quantum states of neutrons in the Earth's gravitational field," Physical Review D, vol. 67, Article ID 102002, 2003.

[4] V. V. Nesvizhevsky, A. K. Petukhov, H. G. Börner et al., "Study of the neutron quantum states in the gravity field," The European Physical Journal C-Particles and Fields, vol. 40, no. 4, pp. 479491, 2005. 
[5] S. Baessler, "Gravitationally bound quantum states of ultracold neutrons and their applications," Journal of Physics G, vol. 36, Article ID 104005, 2009.

[6] V. V. Nesvizhevsky, "Near-surface quantum states of neutrons in the gravitational and centrifugal potentials," Physics-Uspekhi, vol. 53, no. 7, pp. 645-675, 2010.

[7] M. Kreuz, V. V. Nesvizhevsky, P. Schmidt-Wellenburg et al., "A method to measure the resonance transitions between the gravitationally bound quantum states of neutrons in the GRANIT spectrometer," Nuclear Instruments and Methods in Physics Research A, vol. 611, no. 2-3, pp. 326-330, 2009.

[8] T. Jenke, P. Geltenbort, H. Lemmel, and H. Abele, "Realization of a gravity-resonance-spectroscopy technique," Nature Physics, vol. 7, pp. 468-472, 2011.

[9] G. Ichikawa, S. Komamiya, Y. Kamiya et al., "Observation of the spatial distribution of gravitationally bound quantum states of ultracold neutrons and its derivation using the Wigner function," Physical Review Letters, vol. 112, Article ID 071101, 2014.

[10] P. Schmidt-Wellenburg, J. Barnard, P. Geltenbort et al., "Efficient extraction of a collimated ultra-cold neutron beam using diffusive channels," Nuclear Instruments and Methods in Physics Research A: Accelerators, Spectrometers, Detectors and Associated Equipment, vol. 577, no. 3, pp. 623-625, 2007.

[11] J. Barnard and V. Nesvizhevsky, "Analysis of a method for extracting angularly collimated UCNs from a volume without losing the density inside," Nuclear Instruments and Methods in Physics Research A, vol. 591, no. 2, pp. 431-435, 2008.

[12] L. A. Chizhova, S. Rotter, T. Jenke et al., "Vectorial velocity filter for ultracold neutrons based on a surface-disordered mirror system," Physical Review E, vol. 89, Article ID 032907, 2014.

[13] V. V. Nesvizhevsky, A. Y. Voronin, R. Cubitt, and K. V. Protasov, "Neutron whispering gallery," Nature Physics, vol. 6, no. 2, pp. 114-117, 2010.

[14] V. V. Nesvizhevsky, "Polished sapphire for ultracold-neutron guides," Nuclear Instruments and Methods in Physics Research A, vol. 557, no. 2, pp. 576-579, 2006.

[15] V. V. Nesvizhevsky, G. Pignol, K. V. Protasov et al., "Comparison of specularly reflecting mirrors for GRANIT," Nuclear Instruments and Methods in Physics Research A, vol. 578, no. 2, pp. 435-438, 2007.

[16] A. Y. Voronin, P. Froelich, and V. V. Nesvizhevsky, "Gravitational quantum states of Antihydrogen," Physical Review A, vol. 83, Article ID 032903, 2011.

[17] A. Y. Voronin, V. V. Nesvizhevsky, and S. Reynaud, "Whispering-gallery states of antihydrogen near a curved surface," Physical Review A, vol. 85, Article ID 014902, 2012.

[18] G. Dufour, P. Debu, A. Lambrecht, V. V. Nesvizhevsky, S. Reynaud, and A. Yu. Voronin, "Shaping the distribution of vertical velocities of antihydrogen in GBAR," The European Physical Journal C, vol. 74, p. 2731, 2014.

[19] A. Y. Voronin et al., Advances in High Energy Physics, 2014.

[20] G. Chardin, P. Grandemange, D. Lunney et al., "CERN-SPSC2011-029. SPSC-P-342,” Tech. Rep., CERN, Geneva, Switzerland, 2011.

[21] P. Crivelli, "Can we observe the gravitational quantum states of positronium?” Advances in High Energy Physics, 2014.

[22] S. Baessler, M. Beau, M. Kreuz et al., "The GRANIT spectrometer," Comptes Rendus Physique, vol. 12, no. 8, pp. 707-728, 2011.

[23] V. V. Nesvizhevsky, "Gravitational quantum states of neutrons and the new granit spectrometer," Modern Physics Letters A, vol. 27, no. 5, Article ID 1230006, 2012.
[24] S. Baessler et al., Advances in High Energy Physics, 2014.

[25] P. Schmidt-Wellenburg, K. H. Andersen, P. Courtoisa et al., "Ultracold-neutron infrastructure for the gravitational spectrometer GRANIT," Nuclear Instruments and Methods in Physics Research A, vol. 611, no. 2-3, pp. 267-271, 2011.

[26] I. Antoniadis, S. Baessler, O. Bertolami et al., "Workshop GRANIT-2010, 14-19 February 2010, Les Houches, France," Comptes Rendus Physique, vol. 12, no. 8, pp. 703-706, 2011.

[27] I. Antoniadis, S. Baessler, V. V. Nesvizhevsky et al., "Quantum gravitational spectroscopy with ultracold systems," Advances in High Ednergy Physics. In press.

[28] A. E. Meyerovich and A. Stepaniants, "Quantized systems with randomly corrugated walls and interfaces," Physical Review B, vol. 60, no. 12, pp. 9129-9144, 1999.

[29] A. E. Meyerovich and I. V. Ponomarev, "Surface roughness and size effects in quantized films," Physical Review B, vol. 65, Article ID 155413, 2002.

[30] Y. Y. Cheng and A. E. Meyerovich, "Mode coupling in quantized high-quality films," Physical Review B, vol. 73, Article ID 85404, 2006.

[31] J. A. Ogilvy and J. R. Foster, "Rough surfaces: gaussian or exponential statistics?" Journal of Physics D: Applied Physics, vol. 22, no. 9, article 1243, 1989.

[32] A. Fubel, M. Zech, P. Leiderer, J. Klier, and V. Shikin, "Analysis of roughness of Cs surfaces via evaluation of the autocorrelation function," Surface Science, vol. 601, no. 7, pp. 1684-1692, 2007.

[33] J. A. Stroscio and W. J. Kaiser, Eds., Scanning Tunneling Microscopy, vol. 27 of Methods of Experimental Physics, Academic Press, New York, NY, USA, 1993.

[34] R. C. Munoz, G. Vidal, M. Mulsow et al., "Surface roughness and surface-induced resistivity of gold films on mica: application of quantitative scanning tunneling microscopy," Physical Review B, vol. 62 , no. 4686, 2000.

[35] I. Kurganskaya, A. Luttge, and A. R. Barron, The Application of VSI (Vertical Scanning Interferometry) to the Study of Crystal Surface Processes, Connexions, July 2009, http://cnx.org/contents/2515fd85-46bf-42d5-8713-018032a6eb 77@4.

[36] M. Escobar and A. E. Meyerovich, "Applications and identification of surface correlations," http://arxiv.org/abs/1404.1291.

[37] M. Escobar and A. E. Meyerovich, "Beams of gravitationally bound ultracold neutrons in rough waveguides," Physical Review A, vol. 83, Article ID 033618, 2011.

[38] M. Escobar and A. E. Meyerovich, "Quantum transport equation for systems with rough surfaces and its application to ultracold neutrons in quantizing gravity field," Journal of Experimental and Theoretical Physics, to be published.

[39] M. E. Robles, C. A. Gonzalez-Fuentes, R. Henriquez et al., "Resistivity of thin gold films on mica induced by electronsurface scattering: application of quantitative scanning tunneling microscopy," Applied Surface Science, vol. 258, no. 8, pp. 3393-3404, 2012.

[40] J. D. Johnson, S. Krinsky, and B. M. McCoy, "Vertical-arrow correlation length in the eight-vertex model and the low-lying excitations of the X-Y-Z Hamiltonian," Physical Review A, vol. 8, no. 5, pp. 2526-2547, 1973.

[41] M. Escobar and A. Meyerovich, "Quantized ultracold neutrons in rough waveguides: GRANIT experiments and beyond," Advances in High Energy Physics. In press.

[42] H. Cheng and T. T. Wu, "Theory of toeplitz determinants and the spin correlations of the two-dimensional ising model. III," Physical Review, vol. 164, no. 2, pp. 719-735, 1967. 
[43] A. E. Meyerovich and V. V. Nesvizhevsky, "Gravitational quantum states of neutrons in a rough waveguide," Physical Review A, vol. 73, Article ID 063616, 2006.

[44] R. Adhikari, Y. Cheng, A. E. Meyerovich, and V. V. Nesvizhevsky, "Quantum size effect and biased diffusion of gravitationally bound neutrons in a rough waveguide," Physical Review A, vol. 75, Article ID 063613, 2007. 

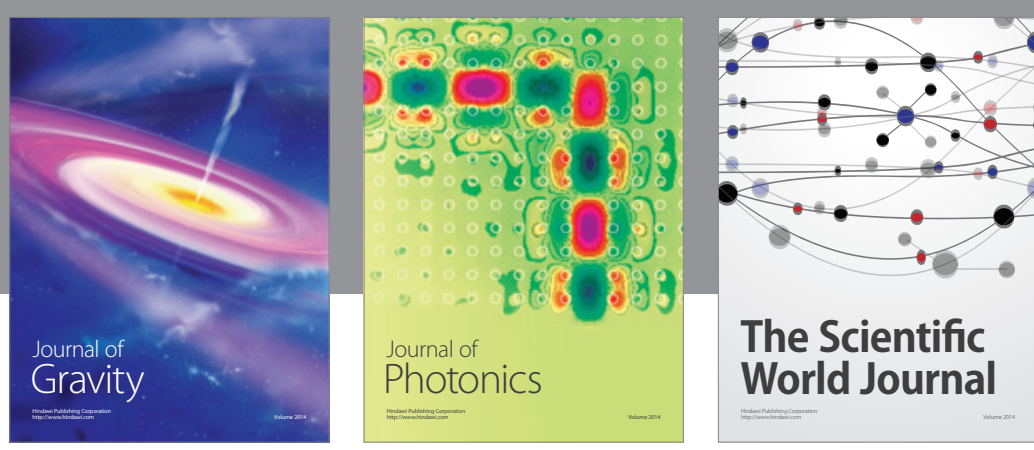

The Scientific World Journal
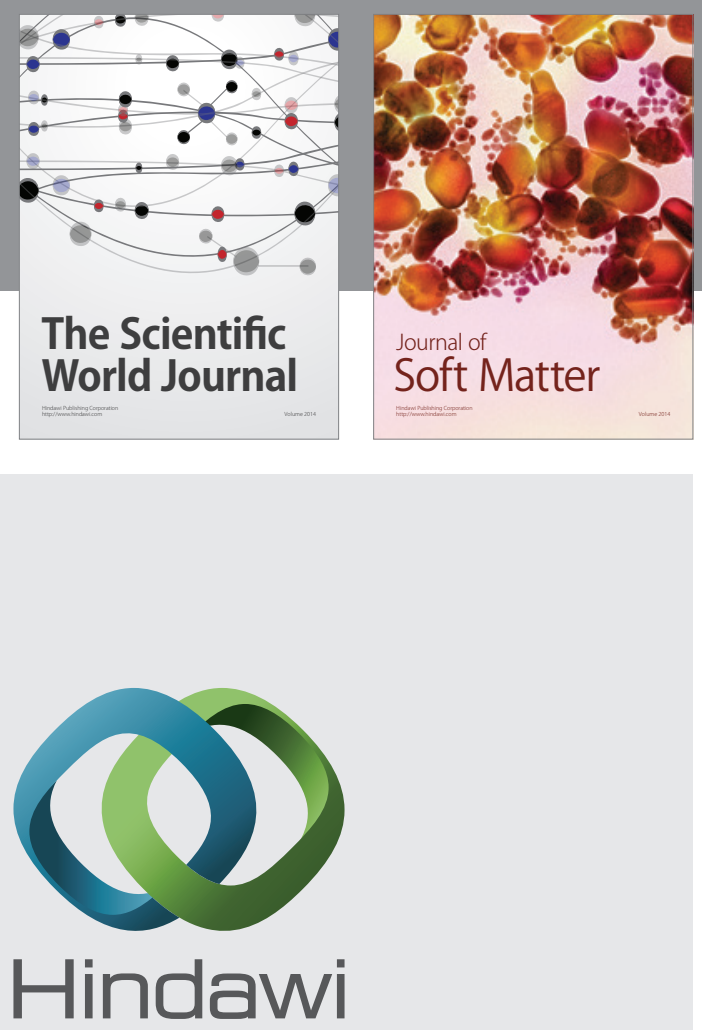

Submit your manuscripts at

http://www.hindawi.com

nternational Journal of

Statistical Mechanics
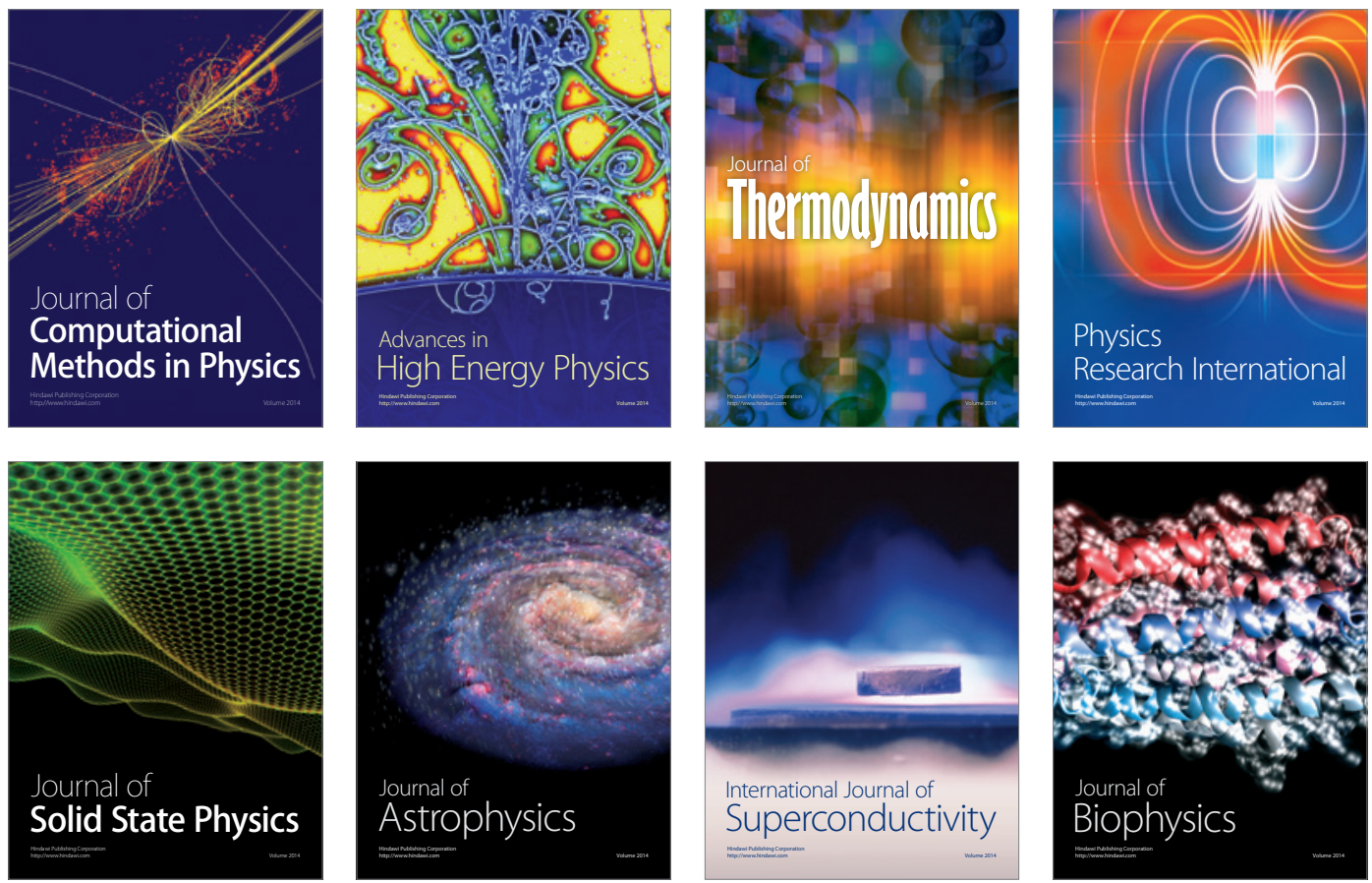
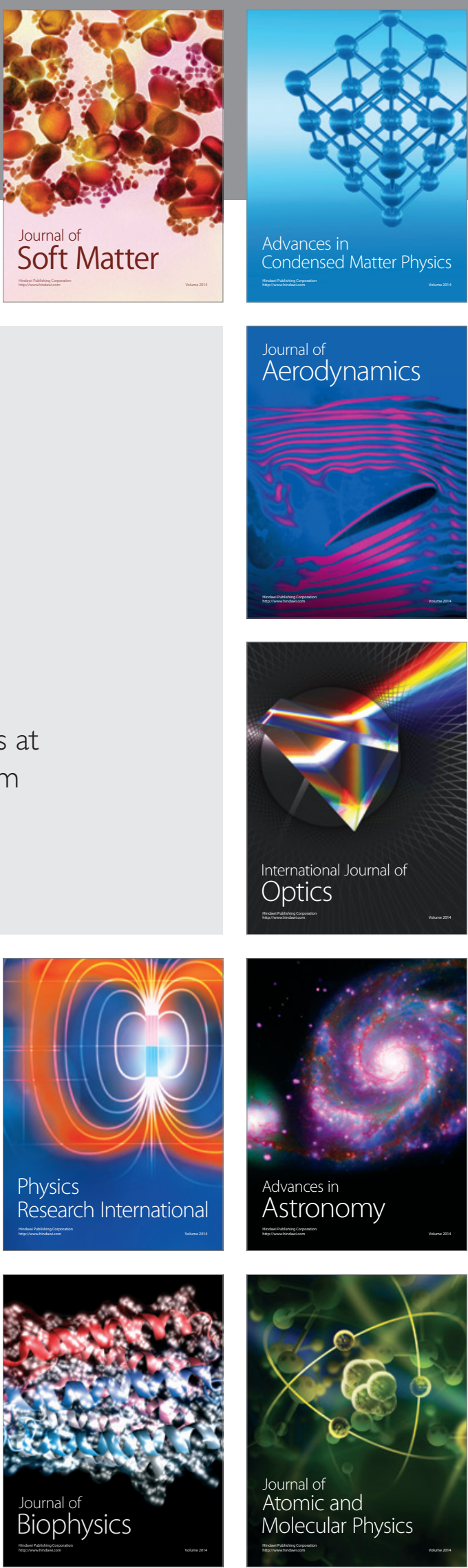